\title{
Le brûlage dirigé pour l'entretien de jeunes peuplements résineux: comportement et impact en fonction de la quantité de litière et de l'élagage
}

\author{
E Rigolot* ${ }^{*}$ JC Valette \\ INRA, station de sylviculture méditerranéenne \\ centre de recherches d'Avignon, Av A Vivaldi, 84000 Avignon, France
}

(Reçu le 22 décembre 1987 ; accepté le 25 septembre 1989)

\begin{abstract}
Résumé - Le bralage dirigé est testé pour l'entretien de plantations de Cupressus arizonica (Green) et de Pinus eldarica (Medw) selon 2 variables, la hauteur d'élagage et la masse de combustible au sol (litiere). En fonction des caractéristiques du feu (hauteur des flammes, vitesse de propagation, puissance dégagée, température atteinte à differents niveaux), son impact sur les arbres est suivi dans le temps au moyen du dessèchement foliaire et des descripteurs de l'état hydrique. Seules les notations du dessèchement foliaire rendent compte de l'impact du feu. Pour Cupressus arizonica, si la charge de combustible au sol ne dépasse pas $5 \mathrm{t} / \mathrm{ha}$ et si les arbres sont élagués au moins sur les premiers $80 \mathrm{~cm}$, alors la pérennité du peuplement est assurée. Pour Pinus eldarica, le feu peut être utilisé jusqu'à une charge de 10 tha sans qu'il ne soit nécessaire d'élaguer; une réduction de la croissance est toutefois constatee.
\end{abstract}

brûlage dirigé / Cupressus arizonica / Pinus eldarica / jeune plantation / dessèchement foliaire / potentiel de sève

Summary - Prescribed burning for cleaning young conifer plantations; behaviour and effects according to the amount of fuel and pruning. The prescribed burning is tested for cleaning Cupressus arizonica (Green) and Pinus eldarica (Medw) plantations following 2 parameters: the pruning level and the amount of fuel on the ground surface (litter). Depending on the fire's components (flame height, spreading rate, power liberated, temperatures reached at different levels), its effect on trees is followed by crown scorch and with hydric status descriptors. Only the crown scorch notations take into account the effects of fire. Under Cupressus arizonica stands, the amount of fuel must not exceed 5 t/ha and the 80 lowest $\mathrm{cm}$ must be pruned, otherwise the trees will be killed. Under Pinus eldarica stands, the fire can be used up to 10 t/ha without any pruning although the growth becomes reduced.

\section{prescribed burning / Cupressus arizonica / Pinus eldarica / young plantation /} crown scorch / sap potential.

\footnotetext{
* Correspondance et tirés à part.
} 


\section{INTRODUCTION}

En région méditerrannéenne, l'élimination, ou au moins la réduction, des strates basses (tapis herbace, strate arbustive, verticilles inférieurs secs) d'une plantation forestiere est un des moyens dont dispose le sylviculteur pour la protéger contre l'incendie.

Le brûlage dirigé est susceptible de supprimer simultanément les produits de l'élagage, les strates herbacée et arbustive ainsi que la couverture morte (Dieterich, 1976 ; Mobley et al, 1978). C'est un feu conduit en hiver en choisissant des conditions de milieux et de végetation telles que les arbres et le sol ne soient pas endommagés et que la progression du feu soit constamment maîtrisée. Cet outil de débroussaillement, pratique et économique, constitue un élement de solution pour l'entretien des pare-feux arborés de certains milieux de la région méditerranéenne (Valette et Maréchal, 1986).

Pour être envisagée, son utilisation pour entretenir les jeunes plantations demande que soit étudiée et quantifiée la réaction des arbres au passage du brûlage dirigé.

Le présent article analyse les caractéristiques techniques du brûlage dirigé à la recule dans une plantation de Cupressus arizonica (Green) et de Pinus eldarica (Medw), et évalue les conséquences du passage du feu sur les arbres selon 2 paramètres :

- le niveau d'élagage compatible avec l'équilibre du végétal (circonférence de la tige, volume du houppier),

- la puissance du feu liée à la masse de combustible au sol.

Plusieurs parametres, descriptifs de l'impact du feu, sont suivis après son passage :

Wyant et al (1986 ; Peterson et Arbaugh (1986) s'accordent à constater que la part de dessèchement foliaire est le meilleur paramètre pour prédire la survie des conifères après des brôlages diriges ou des feux sauvages. Ces auteurs soulignent que ce paramètre traduit en fait une baisse de la capacité photosynthétique des arbres et, par conséquent, un affaiblissement des individus. Ils deviennent alors plus sensibles à la sécheresse ou aux insectes parasites.

Le suivi de paramètres physiologiques permet de faire état de disfonctionnements internes consécutifs au passage du feu, et ceci sans attendre l'apparition des indices externes. Ritchie et Hinckley (1975) montrent que le potentiel de séve est un bon descripteur du comportement hydrique des végétaux. Aussenac et Valette (1982) le confirment sur plusieurs espèces forestières méditerranéennes arborées.

\section{MATÉRIEL ET MÉTHODE}

\section{Le dispositif des Vignères}

Cupressus arizonica et Pinus eldarica présentent l'intérêt d'avoir fait partie des espèces sélectionnées dans les arboretums d'élimination de l'INRA comme essences potentielles de reboisement en région méditerranéennes (Pestour, 1984). De plus, Clément et Caramelle (1978) ont montré que Cupressus arizonica a une inflammabilité moins forte que celles d'especes autochtones, telles que Pinus halepensis ou Quercus ilex. Des études d'inflammabilité sur Pinus eldarica restent à faire.

Les caractéristiques du peuplement expérimental, détaillées par Rigolot (1986), sont reportées au tableau I. Sur les terres agricoles des Vignères les arbres sont bien venants.

Afin d'uniformiser les traitements, les produits de l'élagage, la litiere et la strate herbacée sont remplacées par de la paille de blé. 
Tableau I. Caractéristiques du peuplement experimental des Vignères.

\begin{tabular}{|c|c|}
\hline Localisation & $20 \mathrm{~km}$ au S.E. d'Avignon \\
\hline Date de mise en place & Décembre 1979 \\
\hline Âge depuis la plantation & 6 ans \\
\hline Types de plants & $\begin{array}{l}\text { Cupressus arizonica 1-0 } \\
\text { Pinus eldarica 2-0 }\end{array}$ \\
\hline Densité à la plantation & $\begin{array}{l}40000 \text { tiges/ha } \\
\text { (intermédiaire entre régénération } \\
\text { naturelle et plantation classique) }\end{array}$ \\
\hline Densité après éclaircie (1984) & 20000 tiges $/ \mathrm{ha}$ \\
\hline $\begin{array}{l}\text { Inventaire dendrométrique } \\
\text { (Octobre 1985) }\end{array}$ & $\begin{array}{l}\text { Valeurs pour les } 2 \text { especes: } \\
\text { * Hauteur moyenne }: 4 \mathrm{~m} \\
\text { * Accroissement } \\
\text { moyen en hauteur : } 66 \mathrm{~cm} / \mathrm{an}\end{array}$ \\
\hline
\end{tabular}

L'étude menée par Valette (1986), a conduit à opter pour les valeurs suivantes des paramètres étudiés :

- charges de paille : 500 et $1000 \mathrm{~g} / \mathrm{m}^{2}$;

- niveau d'élagage : 80 et $160 \mathrm{~cm}$

Ces 2 charges correspondent à celles d'un tapis herbacé respectivement moyennement dense et dense (Delaveaud, 1981 ; Maréchal et Valette, 1988). Pour les 2 espèces, 6 placettes d'une trentaine d'individus sont comparées à 1 placette témoin non élaguée et non brúlée. Pour le traitement statistique des données, les individus de la même placette sont considérés comme autant de répétitions du même traitement.

\section{Les brûlages dirigés}

Les brûlages dirigés sont conduits sur les placettes de Cupressus arizonica et sur celles de Pinus eldarica respectivement les 14 et 21 avril 1986.

À ces 2 dates, la température a varié dans des intervalles respectifs de $11,5^{\circ} \mathrm{C}$ à $15,5^{\circ} \mathrm{C}$ et de $16{ }^{\circ} \mathrm{C}$ à $19,5{ }^{\circ} \mathrm{C}$ respectivement et l'humidité de l'air, de $36 \%$ à $52 \%$ et de $51 \%$ à $72 \%$ respectivement.

Des différentes techniques décrites par Delaveaud (1981), celle du feu à la recule est la plus simple, la plus sûre et la moins agressive.
Le feu progresse à contre-vent de sorte que le combustible est préchauffé uniquement par le rayonnement de la base du front de feu tandis que l'autre face du front rayonne sur la zone déjà brûlée, le sol étant protégé par la couche de matières charbonneuses. Ainsi le front de flamme progresse lentement et les flammes couchées par le vent sont de faible hauteur. Les caractéristiques de forme du front de flamme et sa vitesse de progression sont notées tandis que les variations de temperatures au niveau des thermocouples sont enregistrées automatiquement.

\section{Le traitement des données récoltées lors de la progression du feu}

\section{La puissance des feux}

L'équation de Byram (1959) permet le calcul de la puissance linéaire du front de feu, grandeur communément utilisée pour évaluer la sévérité des traitements : $P f=M \times V \times C$ avec $P f=$ puissance du front de feu, en $\mathrm{J} \cdot \mathrm{s}^{-1} \cdot \mathrm{m}^{-1}$

$M=$ masse de combustible effectivement brûlée, en $\mathrm{g} / \mathrm{m}^{2}$;

$V=$ vitesse de propagation du front de feu, en $\mathrm{m} / \mathrm{s}$;

$C=$ pouvoir calorifique inférieur du combustible, en $\mathrm{J} / \mathrm{g}$. 


\section{Les courbes “températures-temps»}

Les températures ont été enregistrées automatiquement aux 6 niveaux suivants : $-2 \mathrm{~cm}$ par rapport au niveau du sol; $0 \mathrm{~cm} ; 40 \mathrm{~cm}$; $80 \mathrm{~cm} ; 120 \mathrm{~cm} ; 160 \mathrm{~cm}$.

Trois arbres par placette, parmi les plus représentatifs, ont été choisis comme supports de 7 séries de thermocouples. Les enregistrements ont permis de tracer les courbes "températures-temps".

L'aire de la zone délimitée par la courbe "températures-temps" et la droite correspondant à 1 seuil de température choisi permet d'indexer la puissance du feu et, plus particulièrement, l'énergie reçue au niveau du point de mesure. Cette grandeur prend en compte la durée pendant laquelle la température est supérieure au seuil de $70{ }^{\circ} \mathrm{C}$, au delà de laquelle Valette (1986) note des dégats irréversibles sur les feuillages de ces mêmes essences.

\section{Comparaison des températures enre- gistrées sur et sous l'écorce}

Les thermocouples de la série $n^{\circ} 5$ sont enfoncés sous l'écorce et enregistrent la température au niveau des cambiums. Ceux de la série $n^{\circ} 3$ sont placés aux mêmes endroits mais sur écorce afin d'en évaluer le pouvoir isolant.

\section{Le suivi à moyen terme : descrip- teurs de l'état hydrique}

Les arbres sont suivis à partir d'un point " 0 ", antérieur au feu, jusqu'à la fin de la période de stress hydrique. L'objectif poursuivi est une discrimination des traitements par les conditions difficiles dues à la sécheresse estivale.

L'état hydrique d'un végétal peut être caractérisé notamment par :

- le potentiel hydrique total, mesuré par le potentiel de sève, avec la «bombe de Scholander" (Scholander et al, 1965 ; Aussenac et Chassagne, 1974) ;

- la turgescence relative et la teneur en eau, 2 paramètres mesurant la quantité d'eau contenue dans un échantillon de végétal.

Cinq arbres par traitement parmi les plus representatifs sont ainsi suivis à un rythme hebdomadaire. Cinq cèdres d'une placette voisine y sont adjoints, leur comportement hydrique est bien connu et sert de référence (Aussenac et Granier, 1978 ; Braesco, 1980 ; Aussenac et Valette, 1982).

\section{Le suivi à long terme : évolution des dessechements foliaires}

Pour suivre l'évolution des dessèchements foliaires, un système de notation analogue à celui employé dans les études de phytotoxicité (CEB, 1966) est adopté (tableau II) ; les notes 3 et surtout 4 et 5 sanctionnent des dégâts irréversibles et préjudiciables à l'avenir de l'arbre.

\section{RÉSULTATS}

Les résultats ci-après sont à analyser en tenant compte, d'une part des limites du dispositif (absence de répétitions des traitements, faibles effectifs) et d'autre part, des différences entre les conditions microclimatiques (tem-

Tableau II. Notation des dessèchements foliaires.

\begin{tabular}{cl}
\hline note & \multicolumn{1}{c}{ Etat des dessechements folaires } \\
\hline 0 & aucun dessèchement foliaire \\
1 & plus de $2 / 3$ de la masse foliaire est vert \\
2 & $1 / 3$ a $2 / 3$ de la masse foliaire totale est vert \\
3 & $1 / 3$ au plus de la masse foliaire totale est vert \\
4 & quelques rameaux verts \\
5 & mort apparente de i'individu \\
\hline
\end{tabular}


pérature et humidité de l'air) régnant sur chacun des 12 feux, tant le 14 que le 21 avril 1986.

\section{Résultats immédiats}

\section{Puissance linéaire du front de feu}

Les puissances calculées sur les placettes à $500 \mathrm{~g} / \mathrm{m}^{2}$ sont assez faibles : $44 \mathrm{~kJ} \cdot \mathrm{m}^{-1} \cdot \mathrm{s}^{-1}$ en moyenne, de l'ordre de celles énoncées par Byram (1959) pour des feux à la recule de graminées. Celles des placettes à $1000 \mathrm{~g} / \mathrm{m}^{2}$ sont en moyenne de $114 \mathrm{~kJ} \cdot \mathrm{m}^{-1} \cdot \mathrm{s}^{-1}$ soit plus du double, en raison de l'effet cumulé de la masse de combustible et de la vitesse de propagation.

\section{Les courbes «températures-temps»}

Les courbes de variation de la température en fonction du temps ont l'allure classique décrite par Trabaud (1979) (fig 1).

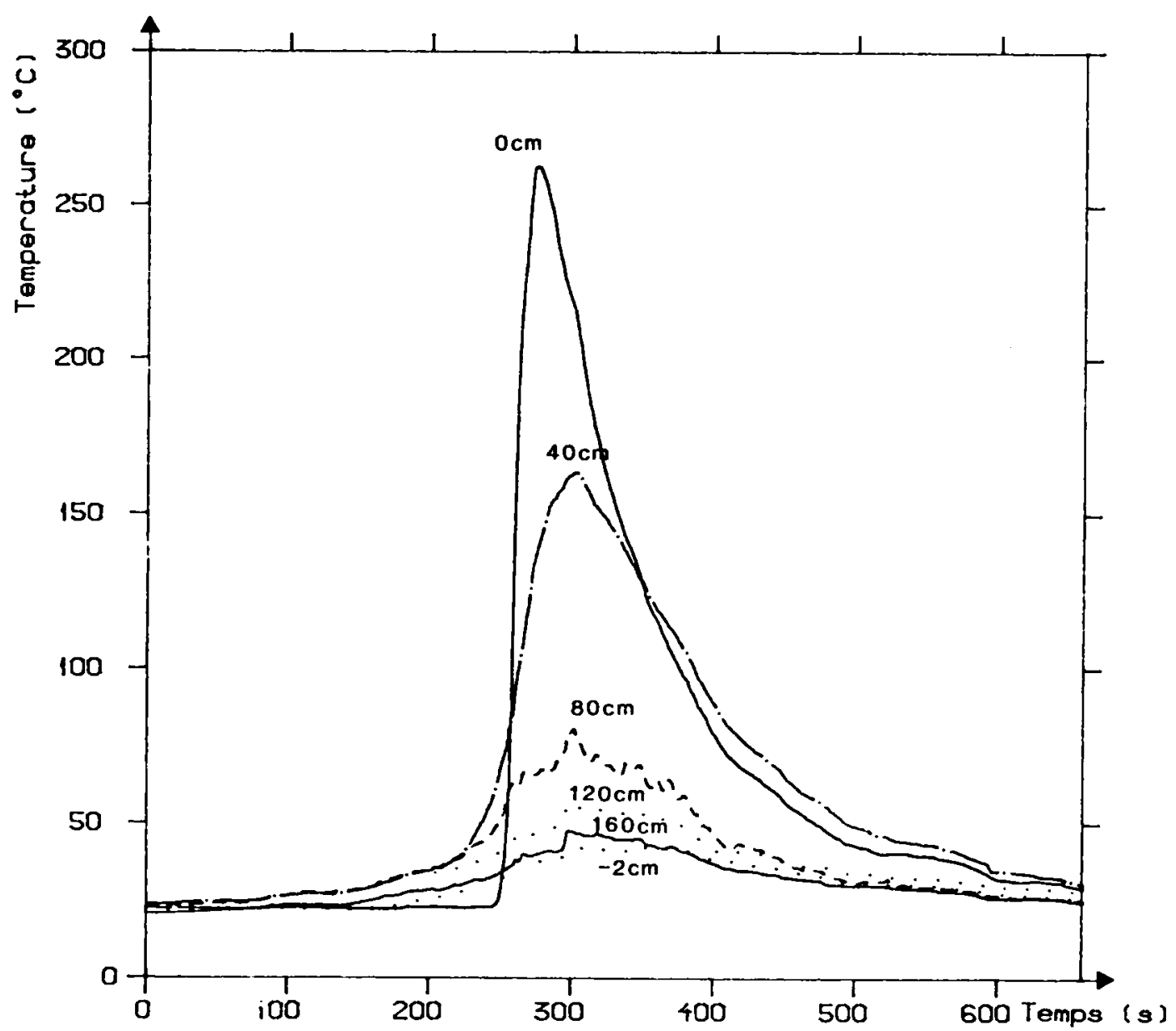

Fig 1. Variation dans le temps de la température de l'air à la surface du tronc et à différents niveaux (Placette : Pinus eldarica, charge $1000 \mathrm{~g} / \mathrm{m}^{2}$, élagage $160 \mathrm{~cm}$ ). 
L'étude des moyennes par niveau et par traitement des températures maximales atteintes permet de mettre en évidence un gradient de hauteur négatif. La comparaison des moyennes de ces valeurs a montré que la zone de température élevée du brâlage dirigé se situe entre 0 et $40 \mathrm{~cm}$, sauf sur les placettes de charge forte et d'élagage faible ou nul, où la limite supérieure de cette zone s'élève à $80 \mathrm{~cm}$.

Les analyses des moyennes des aires au seuil de $70{ }^{\circ} \mathrm{C}$ (tableau III) conduisent aux mêmes conclusions en faisant ressortir les ecarts considera- bles entre les "énergies reçues" au niveau de la base des troncs (niveaux 0 et $40 \mathrm{~cm}$ ) et celles reçues aux niveaux $80 \mathrm{~cm}$ et plus. Cette analyse souligne également la grande dispersion des valeurs. De plus, à température maximale du même ordre, les durées pendant lesquelles les températures sont supérieures à $70{ }^{\circ} \mathrm{C}$, varient considérablement.

Ces résultats invitent à crienter les études futures sur les effets de l'importance et surtout des durées des échauffements subis par la base du tronc de l'arbre.

Tableau III. Quantification de l'échauffement (seuil $70^{\circ} \mathrm{C}$, en ${ }^{\circ} \mathrm{C} . \mathrm{s}$ ).

Remarque : Un mauvais fonctionnement de la centrale d'acquisition de données Orion n'a pas permis l'enregistrement des températures pour la placette de pins $500 \mathrm{~g} / \mathrm{m}^{2}-0 \mathrm{~cm}$.

$N B$ : L'ordre alphabétique fournit le classement, une méme lettre regroupe des moyennes non significativement différentes au seuil de $5 \%$.

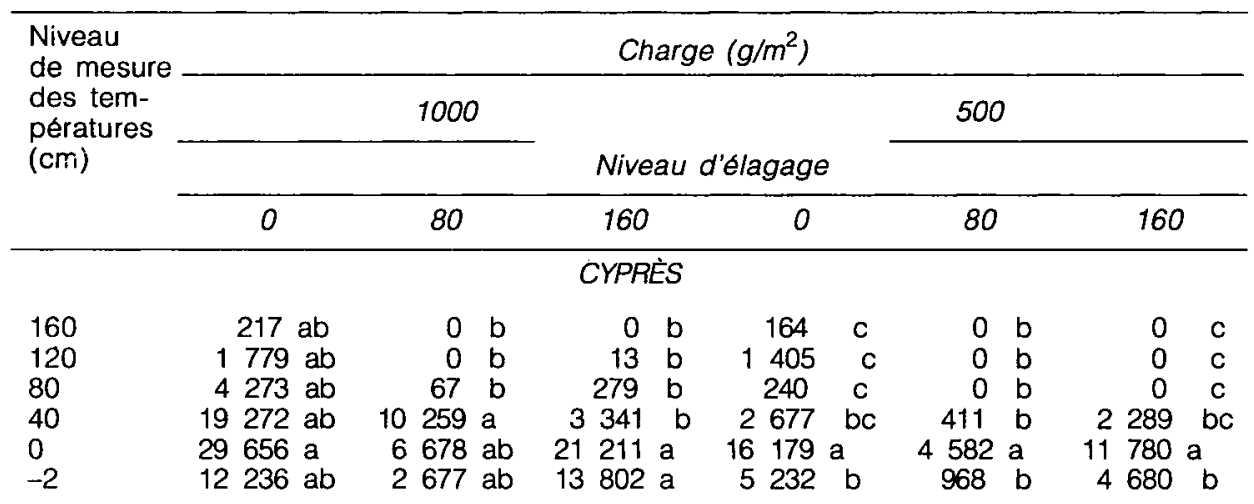

PINS

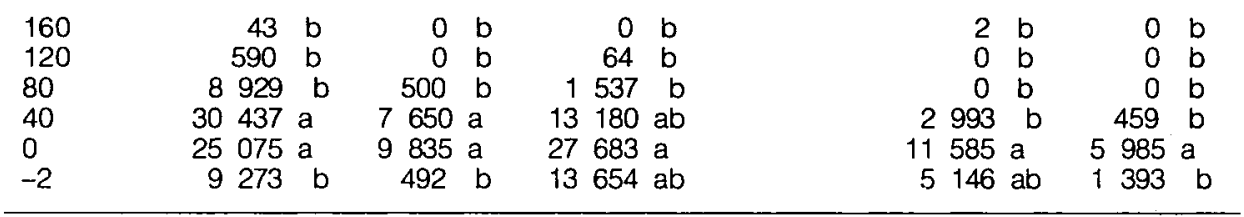


Comparaison des températures "sur" et "sous écorce"

Les oscillations des températures "sous écorce" sont très amorties par rapport à celles enregistrées "sur écorce" (fig 2). L'écart entre ces températures illustre le pouvoir isolant de l'écorce. Cet écart est beaucoup plus important pour Pinus eldarica que pour Cupressus arizonica en raison de la plus grande épaisseur de l'écorce des pins, laquelle varie entre 8 et $12 \mathrm{~mm}$ au niveau du collet, contre une épaisseur de 0,5 à $0,8 \mathrm{~mm}$ à ce même niveau pour les cyprès. Ce caractère "épaisseur de l'écorce au collet", certains auteurs parlent de "densité de l'é-

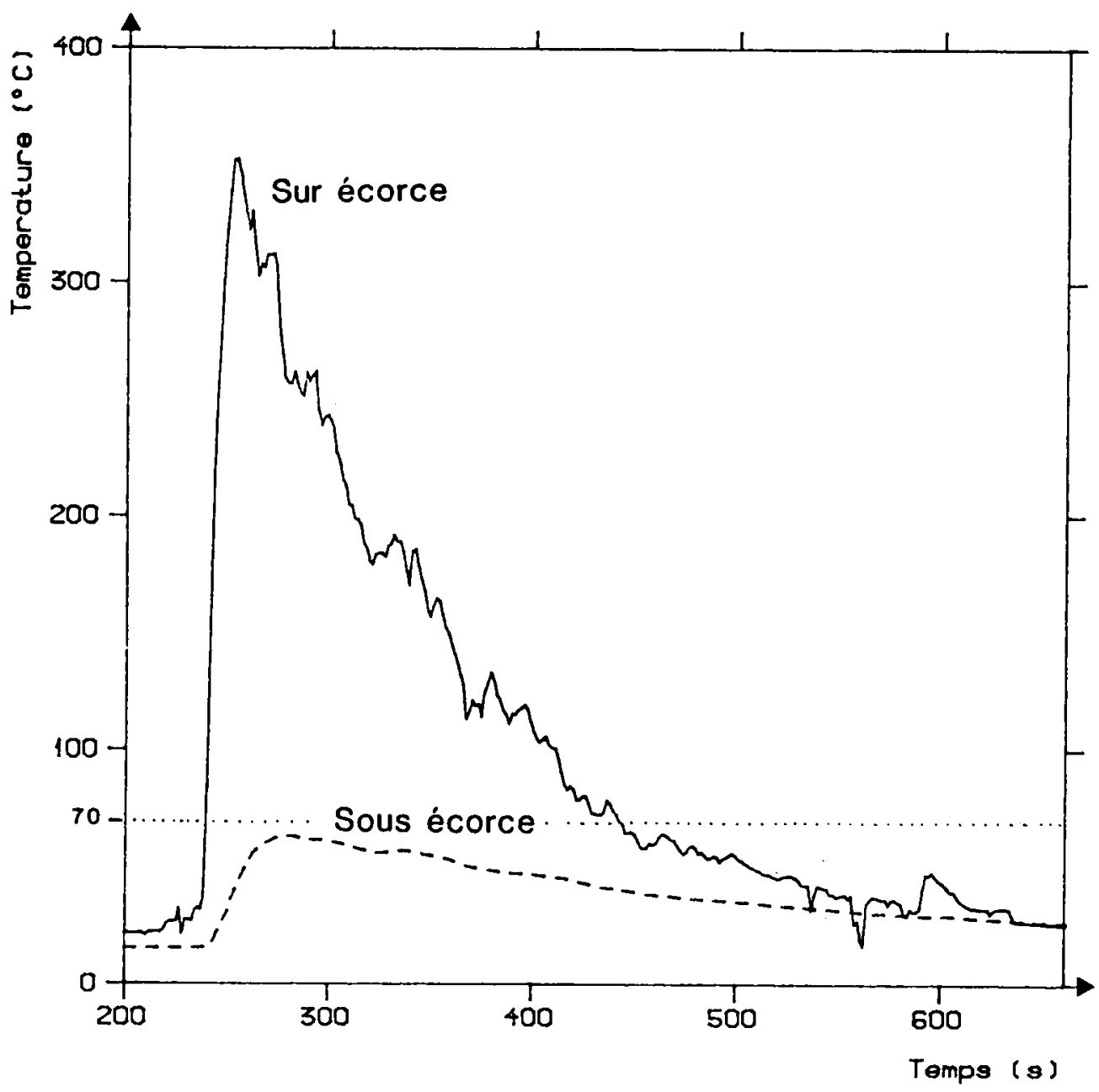

Fig 2. Températures "sur» et "sous" écorce (Placette: Pinus eldarica, charge $1000 \mathrm{~g} / \mathrm{m}^{2}$, élagage $80 \mathrm{~cm}$. Niveau de la mesure : $0 \mathrm{~cm}$ ). 
corce au collet", semble primordial. C'est à ce niveau que les assises cambiales sont soumises aux températures les plus élevées qui peuvent être létales. Ces nécroses peuvent entraîner des annelations partielles plus ou moins importantes qui affaiblissent l'individu et qui sont parfois mortelles pour lui.

\section{Diagnostic après le brôlage}

Dans les conditions expérimentales présentes, les verticilles inférieurs des arbres non élagués constituent un combustible supplémentaire qui augmente la puissance du feu et permet la montée des flammes dans les houppiers. Les dessèchements foliaires, immédiatement après le feu, sont faibles, voire nuls dans les placettes élaguées.

\section{Évolution saisonnière des trois des- cripteurs de l'état hydrique}

Pour chaque traitement, l'évolution saisonnière du potentiel de base, de la turgescence relative et de la teneur en eau, à été suivi.

L'analyse de l'ensemble des placettes ne fait pas apparaître d'effet net des traitements. Le potentiel de base passe par un maximum au début de la période d'étude, les fortes pluies du début avril ont rechargé le sol. Il décroît ensuite jusqu'à un minimum relatif atteint avant l'orage du 17 juin $(32 \mathrm{~mm}$ d'eau). Les réserves en eau du sol sont alors partiellement reconstituées et le potentiel de base remonte nettement. II diminue à nouveau jusqu'à la mi-août et atteint alors un minimum, absolu chez les cèdres et les pins, un minimum relatif voisin du précédent chez les cyprès. La légère remontée de la fin août s'amorce d'une façon inexplicable avant la reprise des précipitations et la baisse de température qui l'accompagne. II apparaît donc clairement que l'orage du 17 juin suspend la sécheresse et modère fortement le stress hydrique.

La turgescence relative et la teneur en eau réagissent beaucoup plus discrètement à cet orage et leurs variations saisonnières sont difficiles à expliquer. Néanmoins les turgescences relatives des pins et des cedres semblent superieures à celles des cyprès. De même, les teneurs en eau des pins et des cedres varient entre $100 \%$ et $200 \%$ alors que celles des cyprès sont plus concentrées autour de $100 \%$.

Quel que soit le descripteur, aucune différence significative au seuil $5 \%$ n'apparaît entre les différents traitements.

Compte tenu de ces constatations, et dans les conditions de l'été 1986 aux Vignères, ces 3 descripteurs ne rendent pas compte des differences d'impact du feu.

\section{Résultats à long terme : évolution des dessechements foliaires}

La figure 3 présente, pour chaque placette, la répartition des individus pour chacune des 5 notes en novembre 1987, soit 19 mois après les feux. Alors qu'au terme de l'été 1986, soit 6 mois après les traitements, les cyprès semblaient avoir mieux résisté au brûlage dirigé que les pins, plus de 1 an après cette constatation, les arbres ont considerablement évolué :

\section{Pour Cupressus arizonica :}

- seules les placettes $500 \mathrm{~g} / \mathrm{m}^{2}$ - $160 \mathrm{~cm}$ et $500 \mathrm{~g} / \mathrm{m}^{2}-80 \mathrm{~cm}$ présentent finalement une mortalité acceptable ;

- dans la placette $500 \mathrm{~g} / \mathrm{m}^{2}$ - $0 \mathrm{~cm}$ près de $20 \%$ des arbres sont morts ou sévèrement endommagés et cette pla- 

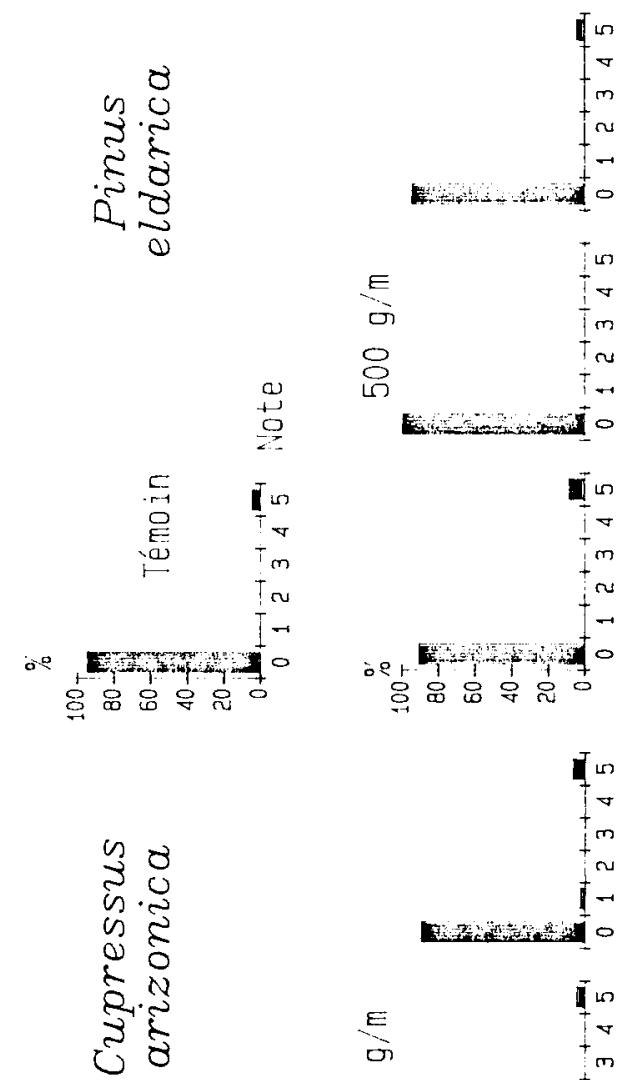

热

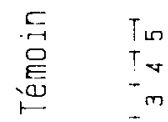

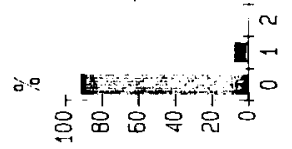

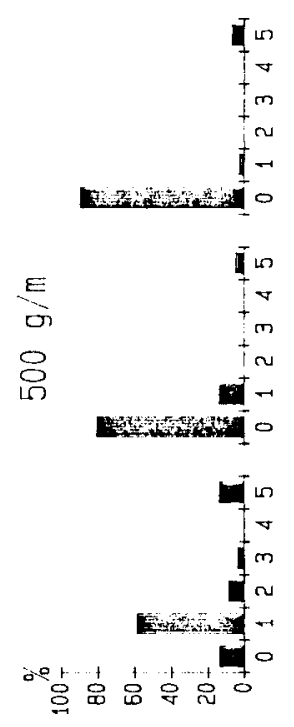

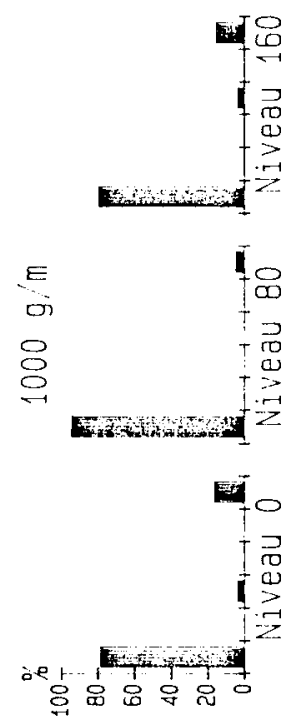

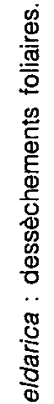
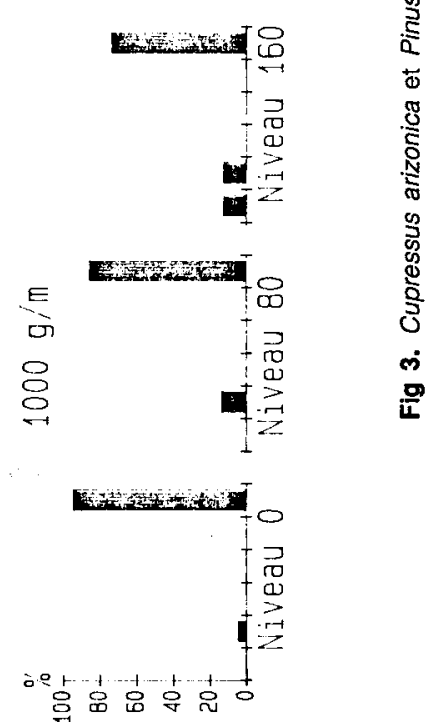
cette constitue une transition avec les placettes traitées à $1000 \mathrm{~g} / \mathrm{m}^{2}$ qui sont toutes très touchées; leurs taux de mortalité s'étalent entre 75 et $95 \%$.

\section{Pour Pinus eldarica :}

- les individus des placettes à $500 \mathrm{~g} / \mathrm{m}^{2}$ ont finalement bien supporté le feu, quelles que soient les hauteurs d'élagage,

- les placettes à $1000 \mathrm{~g} / \mathrm{m}^{2}$ présentent en fin d'étude 2 populations distinctes : d'une part, une forte majorité d'arbres qui ont bien résisté au feu et qui ne présentent plus de dessèchements foliaires, d'autre part $5 \%$ à $16 \%$ d'arbres morts.

Cette mortalité est probablement due aux attaques successives du parasite primaire Dioryctia sy/vestrella et de parasites secondaires, hylésines du pin et pissodes, qui ont envahi le peuplement de pins, profitant de l'affaiblissement des arbres après le feu. Des écoulements de résine, témoins de l'invasion primaire, sont en effet notés en abondance sur le bas des troncs de ces arbres. Les parasites secondaires sont les véritables responsables de la mortalité des arbres, sur lesquels ils laissent de nombreuses galeries et des annelations partielles. Ces insectes parasites avaient déjà été mis en évidence lors de l'étude de l'état initial du peuplement.

Compte tenu des faibles températures enregistrées sous écorce et de la profondeur des galeries observées, il semble que le brûlage dirigé n'ait pas joué le rôle sanitaire escompté. Les insectes ont, non seulement subsité au sein des arbres des placettes traitées, mais ont pu aussi se développer à partir des placettes témoins voisines. Les conclusions des auteurs recommandant de ne pas utiliser le brûlage dirigé sous des peuplements dejà envahis par un parasite (Rego, 1986), se trouvent ici confirmées.

Dix-neuf mois d'observations de l'evolution des dessèchements foliaires conduisent aux conclusions suivantes : - l'utilisation du brûlage dirigé pour entretenir de jeunes peuplements de $\mathrm{Cu}$ pressus arizonica demande que la charge de $500 \mathrm{~g} / \mathrm{m}^{2}$ ne soit pas dépassée et que les arbres soient élagués sur au moins $80 \mathrm{~cm}$;

- pour les jeunes peuplements de Pinus eldarica, le brûlage dirigé peut être utilisé jusqu'à une charge d'au moins $1000 \mathrm{~g} / \mathrm{m}^{2}$ et sans qu'il soit nécessaire d'élaguer au préalable.

\section{DISCUSSION ET CONCLUSIONS}

Si le suivi saisonnier du potentiel de sève est une bonne méthode pour étudier l'état hydrique d'un arbre (Ducrey, 1988), elle ne permet toutefois de prédire sa mort qu'à très court terme. De plus la lourdeur de la méthode conduit à ne suivre qu'un petit échantillon d'individus. II résulte de ces 2 constatations que cette méthode donne une vision à trop court terme et trop partielle de l'évolution de la population d'arbres traités pour être intéressante dans l'étude de l'impact du brûlage dirigé sur la strate arborée.

Par contre, la notation des dessèchements foliaires est suffisamment simple et rapide pour permettre un suivi exhaustif des individus de la population et obtenir ainsi une discrimination fine des traitements. Cette méthode permet aussi de s'affranchir des variations microclimatiques. Comme il s'agit d'une appréciation et non d'une mesure, les opérateurs doivent étalonner leurs notations et réaliser des relevés 
fréquents, surtout lorsque les effectifs des différentes notes varient.

Quoiqu'il en soit, aucune des méthodes employées dans cette étude n'a permis de prévoir le revirement spectaculaire survenu entre l'automne 1986 et l'automne 1987.

L'échauffement lié au brûlage dirigé a fortement roussi les aiguilles des verticilles bas des pins, mais les assises cambiales ont été protégées par l'écorce épaisse. Au contraire, chez les cyprès, le feuillage peu inflammable a été épargné, tandis que leur cambium a été endommagé, ce qui réduit fortement la descente de la sève élaborée. Mais pour les 2 essences, les vaisseaux du xylème plus internes ont été épargnés, permettant toujours la montée de la sève brute. À l'issue de la $1^{\text {ère }}$ saison de végétation, le feuillage des cyprès est encore vert tandis que le reverdissement du houppier des pins n'est que partiel et ne compense pas le roussissement et la chute des aiguilles échauffées. Lors de la $2^{\text {nde }}$ saison de végétation, les bourgeons des pins qui ont en général résisté à l'échauffement, génèrent de nouvelles aiguilles, mais la non-reconstitution des réserves racinaires des cyprès entraîne leur dépérissement.

Pour s'affranchir d'un long suivi, Wyant et al (1986) ont établi des critères simples permettant de prédire, immédiatement après le passage du feu, l'avenir du peuplement : dessèchement foliaire et carbonisation du quadrat le moins touché du tronc. Cette méthode mérite d'être expérimentée sur d'autres essences.

Dans les conditions expérimentales du dispositif des Vignères, Pinus eldarica supporte bien le brûlage dirigé alors que Cupressus arizonica ne le supporte que dans les 2 traitements les plus doux (une puissance de feu cor- respondant à une charge de paille de $5 \mathrm{t} /$ ha pour des hauteurs d'élagage de $160 \mathrm{~cm}$ et $80 \mathrm{~cm}$ ).

Ces conclusions sont à confirmer dans des conditions de milieu plus difficiles. Toutefois, il est important de retenir que Pinus eldarica se prête mieux à l'entretien par le brûlage dirigé que Cupressus arizonica, alors que cette especce de pin, comme d'ailleurs toutes celles de la section Pinus halepensis présente une plus grande sensibilité à l'incendie.

Ces résultats, associés à ceux obtenus sur d'autres dispositifs expérimentaux, dans le taillis vieilli de Quercus pubescens et d'Acer monspessulanum par exemple (Valette et Maréchal, 1986), confirment le fait que la résistance au feu est très liée à l'épaisseur d'écorce sur les premiers dm du tronc, cette épaisseur dépendant, pour certaines espèces, de l'âge de l'individu. Le brûlage dirigé serait donc à réserver pour entretenir des formations riches en individus ayant une écorce basale épaisse.

Des études sont donc à conduire pour mettre en relation l'épaisseur de l'écorce au collet avec l'intensité et la durée d'échauffement à ce niveau. Les seuils létaux ainsi déterminés orienteront le choix de la technique de conduite du brûlage dirigé appropriée au conditions du milieu.

\section{RÉFÉRENCES}

Aussenac G, Chassagne A (1974) Rapport préliminaire sur la mesure du potentiel hydrique de seve dans les rameaux et les feuilles. Doc int (74-2), station de sylviculture et de production, CNRF Nancy, $8 \mathrm{pp}$

Aussenac G, Granier A (1978) Quelques résultats de cinétique journalière du poten- 
tiel de sève chez les arbres forestiers. Ann Sci For 35, 19-32

Aussenac G, Valette JC (1982) Comportement hydrique estival de Cedrus atlantica Mann, Quercus ilex L et Quercus pubescens Willd et de divers pins dans le Mont-Ventoux. Ann Sci For 39, 41-62

Braesco A (1980) Potentiels de sève et réactions à la sécheresse des cedres, pins et chênes en Provence calcaire (été 1980). Doc int (81-4) INRA, station de sylviculture méditerranéenne, Avignon, $29 \mathrm{pp}$

Byram GM (1959) Combustion of forest fuels. In: Forest Fire : Control and use. (KP Davis ed) Mac Graw-Hill, New York, pp 61-89

CEB (1966) Principes géneraux d'études de l'efficacité $d$ 'herbicides et de la phytotoxicité d'un produit en sylviculture. Comm Essais Biol Phytiatrie Phytopharm, 25, Paris, $26 \mathrm{pp}$

Clément A, Caramelle P (1978) Inflammabilité et combustibilité de la végétation méditerranéenne. INRA station de sylviculture méditerranéenne, Avignon, Mémoire de fin d'études ENITEF, $168 \mathrm{pp}$

Delaveau DP (1981) Le feu, outil sylvicole? Utilisation pratique des données de combustibilité. INRA station de sylviculture méditerranéenne Avignon, Mémoire de $3^{e}$ année ENITEF, $92 \mathrm{pp}$

Dieterich FH (1976) Prescribed burning in Ponderosa pine - State of art. Region 6. Eastside Prescribed Fire Workshop. Bend Oregon

Ducrey M (1988) Réactions à la sècheresse de quelques especes forestières méditerranéennes. Rev For Fr 5, 359-370

Maréchal J, Valette J (1988) Les dispositifs de la Roque d'Anthéron. In : Actes de l'atelier international sur le brolage contrôlé, Avignon, 189-206

Mobley HE et al (1978) A guide for prescribed fire in southern forests. USDA For Serv, Southeastern area, Atlanta, Georgia, USA, $40 \mathrm{pp}$
Pestour JL (1984) Choix des espèces de reboisement en région méditerranéenne : Premier bilan des arboretums d'élimination. INRA station de sylviculture méditerranéenne, Avignon. Mémoire $3^{\theta}$ années ENITEF, $77 \mathrm{pp}$

Peterson DL, Arbaugh JM (1986) Postfire survival in Dougals-fir and Lodgepole pine: comparing the effects of crown and bole damage. Can J For Res, 16, 1 175-1 179

Rego $F$ (1986) Effects of prescribed fire on vegetation and soil properties in Pinus pinaster of Northern Portugal. Thèse Univ Idaho, $108 \mathrm{pp}$

Rigolot E (1986) Impacts du feu contrôlé sur Cupressus arizonica et Pinus eldarica. INRA station de sylviculture méditerranéenne, Avignon, Mémoire $3^{\mathbf{e}}$ années ENSA Rennes, $28 \mathrm{pp}$

Ritchie GA, Hinckley TM (1975) The pressure chamber as an instrument for ecological research. Adv Ecol Res 9, 165-254

Scholander et al (1965) Sap pressure in vascular plants. Science 143, 339-346

Trabaud L (1979) Étude du comportement du feu dans la garrigue de chêne kermès à partir des temperatures et des vitesses de propagation. Ann Sci For 36 13-38

Valette JC (1986) Sensibilité au feu d'espèces forestières méditerranéennes en fonction de la hauteur d'élagage et de la charge de litiere. INRA, Station de Sylviculture Méditerranéenne, Avignon, Communication $18^{\circ}$ Congrès Mondial de I'IUFRO, Ljubljana, $7 \mathrm{pp}$

Valette JC, Maréchal J (1986) Entretien de pare-feu. Bilan 1984-1986. INRA, station de sylviculture méditerranéenne, Avignon, Doc int (17-86), $73 \mathrm{pp}$

Wyant JG, Omi PN, Laven RD (1986) Fire induced tree mortality in a Colorado Ponderosa pine/Douglas-fir stand. For Sci 32, 49-59 